CRYSTALLOGRAPHIC COMMUNICATIONS

ISSN 2056-9890

Received 12 April 2017

Accepted 22 April 2017

Edited by C. Rizzoli, Universita degli Studi di Parma, Italy

₹ Additional corresponding author, email: bk_jeanjules@yahoo.fr.

Keywords: crystal structure; pentacyclic triterpene; quafrinoic acid; Nauclea pobeguiniia; Hirshfeld surface analysis.

CCDC reference: 1545425

Supporting information: this article has supporting information at journals.iucr.org/e

\section{Crystal structure and Hirshfeld surface analysis of 3-oxours-12-ene-27a,28-dioic acid (quafrinoic acid)}

\author{
Jean Jules Bankeu Kezetas, ${ }^{\mathrm{a}} \neq$ Stéphanie Dietagoum Madjouka, ${ }^{\mathrm{b}}$ Rajesh Kumar, \\ Muhammad Shaiq Ali, ${ }^{\mathrm{c}}$ Bruno Lenta Njakou ${ }^{\mathrm{d}}$ and Sammer Yousuf ${ }^{\mathrm{C} *}$
}

a'Department of Chemistry, Faculty of Science, The University of Bamenda, PO Box 39, Bambili, Cameroon, ${ }^{\mathbf{b}}$ Department
of Chemistry, Faculty of Science, University of Yaoundé I, PO Box 812, Yaoundé, Cameroon, ' ${ }^{\text {H.E.J. Research Institute of }}$
Chemistry, International Center for Chemical and Biological Sciences, University of Karachi, Karachi 75270, Pakistan,
and ${ }^{\mathbf{d}}$ Department of Chemistry, Higher Teacher Training College, University of Yaounde I, PO Box 47 Yaoundé,
Cameroon. *Correspondence e-mail: dr.sammer.yousuf@gmail.com

The title compound, $\mathrm{C}_{30} \mathrm{H}_{44} \mathrm{O}_{5}$, is a pentacyclic triterpene isolated from the Cameroonian medicinal plant Nauclea Pobeguinii and known as quafrinoic acid. The molecule is composed of five fused six-membered rings, four of which adopt a chair conformation and one a half-chair conformation. Intramolecular $\mathrm{C}-$ $\mathrm{H}$. . O hydrogen-bond interactions exist, which generate $S 6$ and $S 8$ rings. In the crystal, molecules are linked by pairs of $\mathrm{O}-\mathrm{H} \cdots \mathrm{O}$ hydrogen bonds, linking $R_{2}^{2}(8)$ rings into chains running parallel to the $a$ axis; these chains are further connected into layers parallel to the $a b$ plane by $\mathrm{C}-\mathrm{H} \cdots \mathrm{O}$ hydrogen bonds. The Hirshfeld surface analysis of the crystal structure indicates that the most important contributions for the crystal packing are from $\mathrm{H} \cdots \mathrm{H}(79.4 \%)$ and $\mathrm{O} \cdots \mathrm{H}(20.4 \%)$ interactions.

\section{Chemical context}

Nauclea is a well-known genus of the Rubiaceae family consisting of 35 species of which ten are distributed in tropical Africa, Asia and Australia (Chen \& Taylor, 2011). Several specimens of this genus, including Nauclea pobeguinii, are largely used in traditional medicine in Africa. During the last decade, many studies have been carried out on $N$. pobeguinii to explore its medicinal potential and promising results have made it an attractive target for researchers. The $80 \%$ ethanolic stem bark extract of $N$. pobeguinii has been successfully used in clinical trials for the treatment of uncomplicated malaria (Mesia et al., 2012). The plant is also reported to have cytotoxic, anti-cancer (Kuete et al., 2015) and anti-diabetic properties (Agnaniet et al., 2016). The phytochemical investigations of $N$. pobeguinii have led to the isolation of monoterpene indole alkaloids, triterpenes and phenolic compounds (Kuete et al., 2015; Xu et al., 2012; Zeches et al., 1985). In a continuation of our phytochemical investigation of Cameroonian medicinal plants, we have examined the stem bark of $N$. pobeguinii and isolated quafrinoic acid. Although the atomic connectivity of quafrinoic acid has already been determined by spectroscopic methods (Ajaiyeoba \& Krebs, 2003), we report herein the single crystal X-ray diffraction structure and Hirshfeld surface analysis of quafrinoic acid for 
the first time.

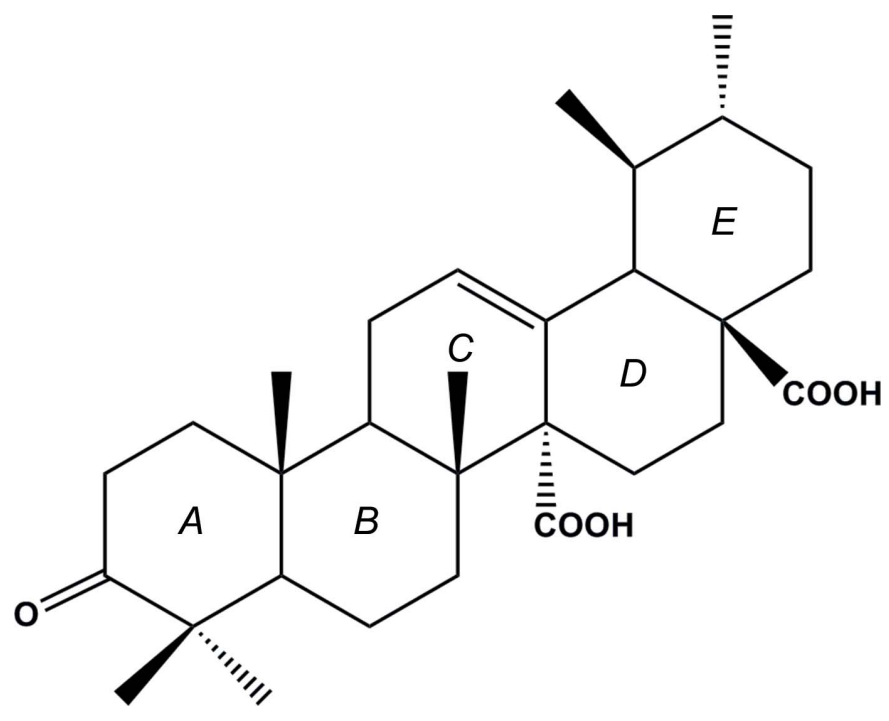

\section{Structural commentary}

The title compound $\mathrm{C}_{30} \mathrm{H}_{44} \mathrm{O}_{5}$, is a pentacyclic triterpene composed of five fused six-membered rings $A$ (C1-C5/C10), $B$ (C5-C10), C (C8-C9/C11-C14), D (C14-C18) and $E$ (C17C18/C25-C28) (Fig. 1). Rings $A, B, D$ and $E$ each exhibit a chair conformation, whereas ring $C$ has a half-chair conformation. Rings $A / B, B / C$ and $C / D$ are trans fused to each other along the $\mathrm{C} 5-\mathrm{C} 10, \mathrm{C} 8-\mathrm{C} 9$, and $\mathrm{C} 13-\mathrm{C} 14$ bonds, respectively. Rings $D$ and $E$ are cis fused along the $\mathrm{C} 17-\mathrm{C} 18$ bond along with the axially oriented carboxylic acid functionalities at $\mathrm{C} 14$ and $\mathrm{C} 17$. The bond dimensions are similar to those found in structurally related compounds (Csuk et al., 2015; Awang et al., 2009).

The molecular conformation is stabilized by intramolecular hydrogen-bonding interactions involving as acceptors the oxygen atoms of the axially oriented carboxylic group $\mathrm{O} 2 / \mathrm{O} 3 /$ $\mathrm{C} 19$ via $\mathrm{C} 7-\mathrm{H} 7 A \cdots \mathrm{O} 3, \quad \mathrm{C} 9-\mathrm{H} 9 A \cdots \mathrm{O} 3$ and $\mathrm{C} 30-$ $\mathrm{H} 30 A \cdots \mathrm{O} 3$ hydrogen bonds and forming rings with $S(6), S(6)$ and $S(8)$ graph-set motifs, respectively (Table 1 ).

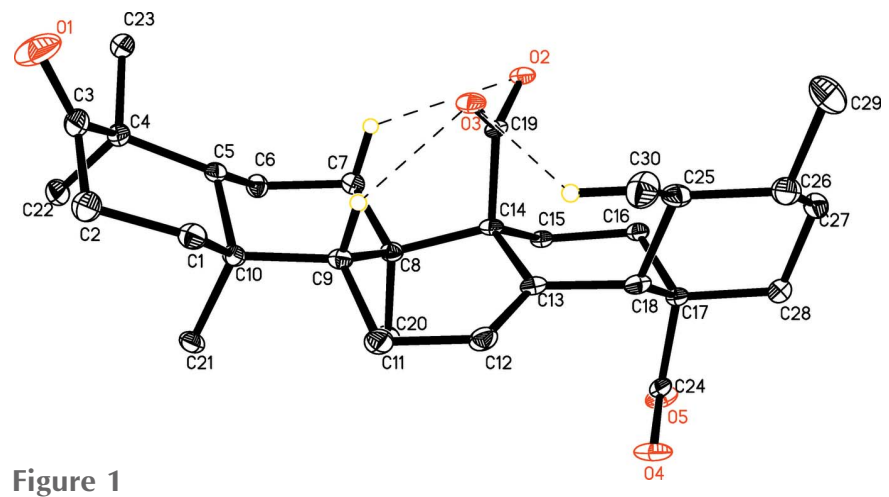

The molecular structure of the title compound, with displacement ellipsoids drawn at the $30 \%$ probability level. Dashed lines indicate intramolecular hydrogen bonds. $\mathrm{H}$ atoms not involved in hydrogen bonding have been omitted.
Table 1

Hydrogen-bond geometry $\left(\AA{ }^{\circ}\right)$.

\begin{tabular}{lllll}
\hline$D-\mathrm{H} \cdots A$ & $D-\mathrm{H}$ & $\mathrm{H} \cdots A$ & $D \cdots A$ & $D-\mathrm{H} \cdots A$ \\
\hline $\mathrm{O} 4-\mathrm{H} 4 A \cdots \mathrm{O} 3^{\mathrm{i}}$ & $0.91(1)$ & $1.70(1)$ & $2.6046(18)$ & $172(5)$ \\
$\mathrm{O} 2-\mathrm{H} 19 A \cdots \mathrm{O} 5^{\text {ii }}$ & $0.80(4)$ & $1.89(4)$ & $2.6702(18)$ & $165(4)$ \\
$\mathrm{C} 7-\mathrm{H} 7 A \cdots \mathrm{O} 2$ & 0.99 & 2.54 & $3.232(2)$ & 127 \\
$\mathrm{C} 9-\mathrm{H} 9 A \cdots \mathrm{O} 3$ & 1.00 & 2.18 & $3.009(2)$ & 139 \\
$\mathrm{C} 28-\mathrm{H} 28 B \cdots \mathrm{O} 1^{\mathrm{iii}}$ & 0.99 & 2.49 & $3.477(3)$ & 173 \\
$\mathrm{C} 29-\mathrm{H} 29 A \cdots \mathrm{O} 4^{\text {iv }}$ & 0.98 & 2.57 & $3.497(3)$ & 158 \\
$\mathrm{C} 30-\mathrm{H} 30 A \cdots \mathrm{O} 3$ & 0.98 & 2.58 & $3.221(3)$ & 123 \\
\hline
\end{tabular}

Symmetry codes: (i) $x+1, y, z ; \quad$ (ii) $\quad x-1, y, z ; \quad$ (iii) $\quad x+1, y-1, z ; \quad$ (iv) $-x+2, y-\frac{1}{2},-z+1$.

\section{Supramolecular features}

In the crystal, molecules are linked into chains parallel to the $a$ axis through pairs of $\mathrm{O}-\mathrm{H} \cdots \mathrm{O}$ hydrogen bonds, forming $R_{2}^{2}(8)$ rings. These chains are further connected into layers parallel to the $a b$ plane by $\mathrm{C}-\mathrm{H} \cdots \mathrm{O}$ hydrogen bonds (Table 1 ; Fig. 2).

\section{Hirshfeld surface analysis}

An Hirshfeld surface analysis (Hirshfeld, 1977; Spackman \& Jayatilaka, 2009) of the title compound was carried out (Fig. 3) to investigate the location of atoms with potential to form hydrogen bonds and the quantitative ratio of these interactions. The analysis of the crystal structure suggests that the most important interaction is $\mathrm{H} \cdot \cdots \mathrm{H}$ contributing $79.4 \%$ to the overall crystal packing. The other important interaction is $\mathrm{O} \cdots \mathrm{H}$, contributing $20.4 \%$ towards the crystal packing. The

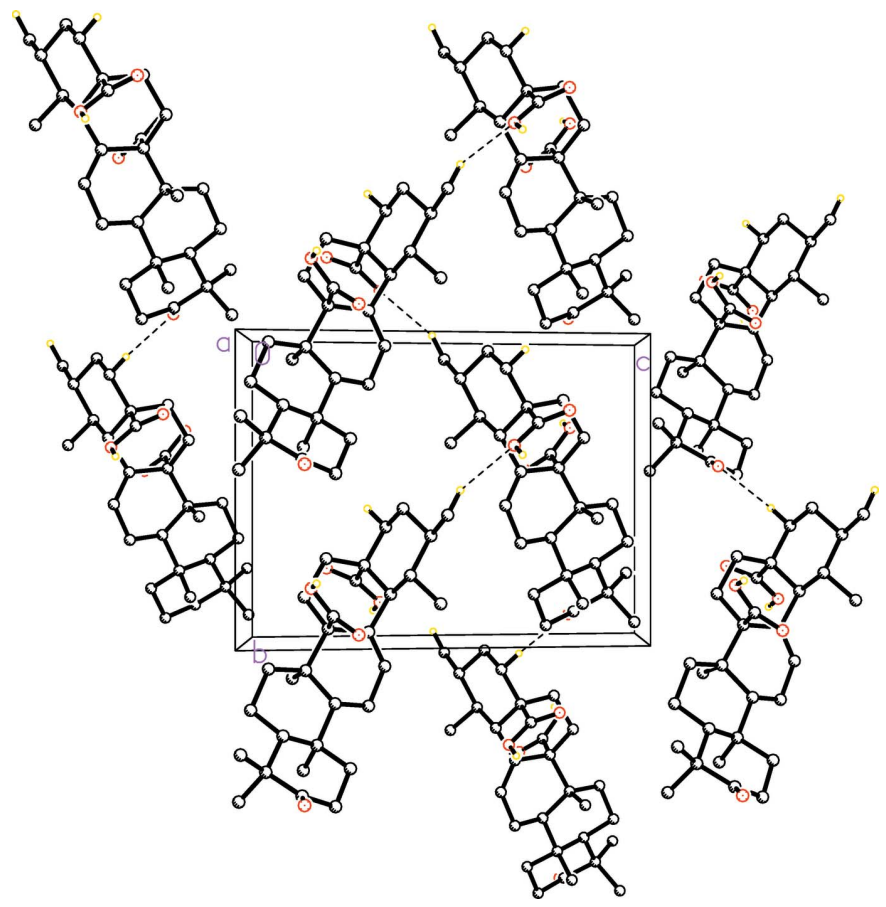

Figure 2

The crystal packing of the title compound viewed down the $a$ axis. Only $\mathrm{H}$ atoms involved in hydrogen bonding are shown. 


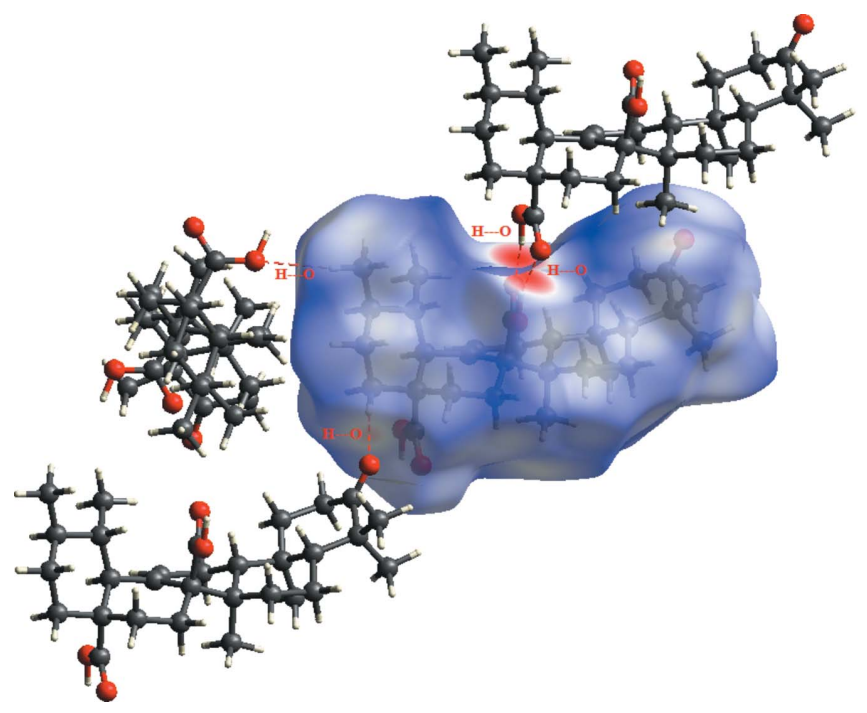

Figure 3

$d_{\text {norm }}$ mapped on Hirshfeld surface for visualizing the inter-contacts of the title compound. Dashed lines indicate hydrogen bonds.

weakest intermolecular contact for the cohesion of the structure is $\mathrm{O} \cdots \mathrm{O}$, found to contribute only $0.4 \%$. The graphical representation of the Hirshfeld surface (Fig. 4) suggests the locations of intermolecular contacts. These contacts are represented by conventional mapping of $d_{\text {norm }}$ on molecular Hirshfeld surfaces as shown in Fig. 3 . The H. .H contribution
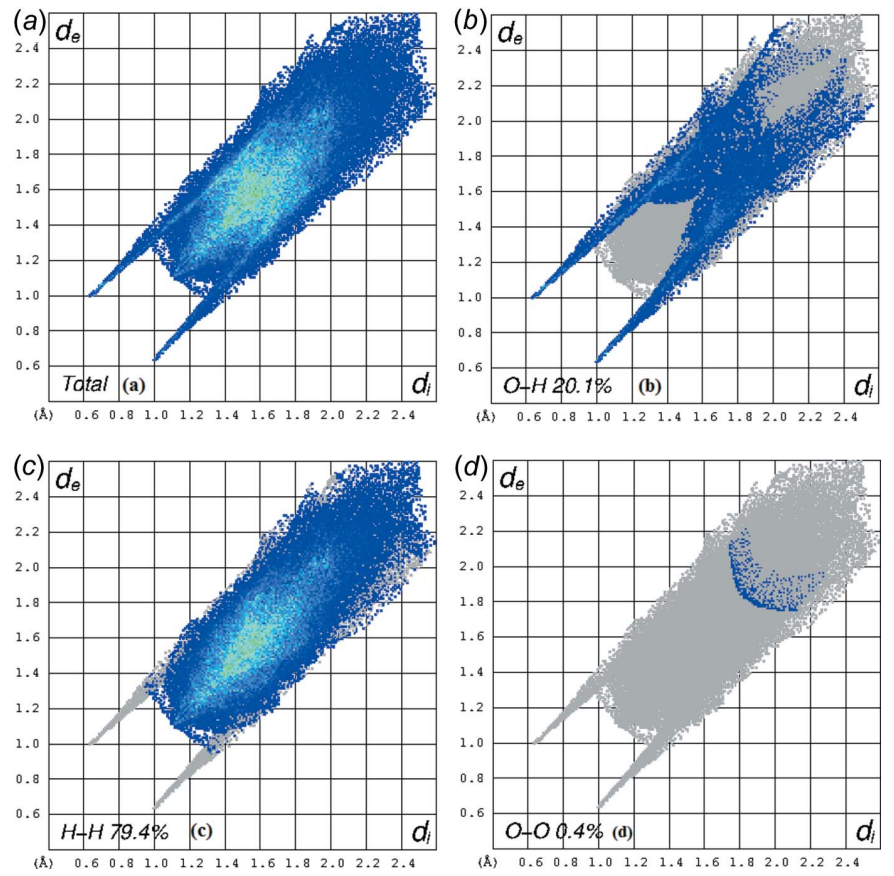

Figure 4

Two-dimensional fingerprint plot analysis of (a) all interactions, $(b)$ $\mathrm{H} \cdots \mathrm{H}$ contacts, $(c) \mathrm{O} \cdots \mathrm{H}$ contacts and $(d) \mathrm{O} \cdots \mathrm{O}$ contacts. The outline of the full fingerprint plots is shown in grey. $d_{\mathrm{i}}$ is the closet internal distance from a given point on the Hirshfeld surface and $d_{\mathrm{e}}$ is the closest external contact.
Table 2

Experimental details.
Crystal data

Chemical formula

$M_{\mathrm{r}}$

Crystal system, space group

$a, b, c(\AA)$

$\beta\left({ }^{\circ}\right)$

$V\left(\AA^{3}\right)$

$Z$

Radiation type

$\mu\left(\mathrm{mm}^{-1}\right)$

Crystal size (mm)

Data collection

Diffractometer

Absorption correction

$T_{\min }, T_{\max }$

No. of measured, independent and observed $[I>2 \sigma(I)]$ reflections

$R_{\text {int }}$

$(\sin \theta / \lambda)_{\max }\left(\AA^{-1}\right)$

Refinement

$R\left[F^{2}>2 \sigma\left(F^{2}\right)\right], w R\left(F^{2}\right), S$

No. of reflections

No. of parameters

No. of restraints

$\mathrm{H}$-atom treatment

$\Delta \rho_{\max }, \Delta \rho_{\min }\left(\mathrm{e} \AA^{-3}\right)$

Absolute structure

Absolute structure parameter
Temperature (K)
$\mathrm{C}_{30} \mathrm{H}_{44} \mathrm{O}_{5}$

484.65

Monoclinic, $P 2_{1}$

100

8.3465 (2), 10.9783 (3), 14.6583 (4)

$101.056(1)$

$\mathrm{Cu} K \alpha$

0.64

$0.45 \times 0.23 \times 0.12$

Bruker SMART APEX CCD areadetector

Multi-scan (SADABS; Bruker, 2009)

$0.760,0.927$

$28696,5116,5023$

0.042

0.618

$0.041,0.115,1.06$

5116

325

2

$\mathrm{H}$ atoms treated by a mixture of independent and constrained refinement

$0.70,-0.31$

Flack, 1983

0.14 (17)
$1318.22(6)$

Computer programs: SMART and SAINT (Bruker, 2009), SHELXTL (Sheldrick, 2008), PLATON (Spek, 2009) and publCIF (Westrip, 2010).

for the crystal packing is shown as a Hirshfeld surface twodimensional fingerprint plot with red dots (Wolff et al., 2012). The $d_{\mathrm{e}}\left(y\right.$ axis) and $d_{\mathrm{i}}(x$ axis) values are the closest external and internal distances $(\AA)$ from a given points on the Hirshfeld surface contacts (Fig. 4).

\section{Synthesis and crystallization}

The stem bark of N. pobeguinii (Pobég. ex Pellegr.) Merr. ex E.M.A., Rubiaceae, were collected in March 2015 from Makénéné, Centre Region of Cameroon, identified by Dr Njouonkou André Ledoux and Mr Tacham Walter Ndam, lecturers in botany at the Department of Biological Sciences, Faculty of Science, The University of Bamenda, and compared with voucher specimens formerly kept at the National Herbarium under the registration number (32597/HNC). $7.2 \mathrm{~kg}$ of the air-dried and ground stem bark of $N$. pobeguinii was extracted with $\mathrm{MeOH}(3 \times 20 \mathrm{~L})$ at room temperature and allowed to concentrate under reduced pressure at low temperature to obtain $1000 \mathrm{~g}$ of brown crude extract. The extract was subjected to medium pressure liquid column chromatography over silica gel (Merck, 230-400 mesh) eluting with $n$-hexane, $n$-hexane/EtOAc, EtOAc and EtOAc/MeOH, in increasing order of polarity to yield quafrinoic acid (25 mg). 
The purified compound was recrystallized by slow evaporation of a methanol solution at room temperature.

\section{Refinement}

Crystal data, data collection and structure refinement details are summarized in Table 2. $\mathrm{H}$ atoms on methyl, methylene and methine carbon atoms were positioned geometrically with $\mathrm{C}-$ $\mathrm{H}=0.96 \AA\left(\mathrm{CH}_{3}\right), 0.97 \AA\left(\mathrm{CH}_{2}\right)$ and $0.93 \AA(\mathrm{CH})$ and constrained to ride on their parent atoms with $U_{\text {iso }}(\mathrm{H})=$ $1.2 U_{\text {eq }}(\mathrm{C})$ or $1.5 U_{\text {eq }}(\mathrm{C})$ for methyl $\mathrm{H}$ atoms. The carboxy $\mathrm{H}$ atoms were located in a difference-Fourier map and refined isotropically, with the $\mathrm{O} 4-\mathrm{H} 4$ bond length constrained to be 0.90 (1) ^.

\section{Acknowledgements}

BJKK is very grateful to The World Academy of Sciences (TWAS) and the International Center for Chemical and Biological Sciences (ICCBS), University of Karachi, Pakistan for their financial and technical support through the ICCBSTWAS Postdoctoral Fellowship number 3240280476 granted to BKJJ.

\section{References}

Agnaniet, H., Mbot, E. J., Keita, O., Fehrentz, J.-A., Ankli, A., Gallud, A., Garcia, M., Gary-Bobo, M., Lebibi, J., Cresteil, T. \& Menut, C. (2016). BMC Complement. Altern. Med. 16, 71-78.
Ajaiyeoba, E. O. \& Krebs, H. C. (2003). Nigerian J. Nat. Prod. Med. 7, 39-41.

Awang, K., Abdullah, N. H., Thomas, N. F. \& Ng, S. W. (2009). Acta Cryst. E65, o2113.

Bruker (2009). SADABS, SMART and SAINT. Bruker AXS Inc., Madison, Wisconsin, USA.

Chen, T. \& Taylor, C. M. (2011). Nauclea linnaeus, sp. P1, in Flora of China, edited by Z.-Y. Wu, P. H. Raven \& D.-Y. Hong, Vol. 19, p. 249. Beijing: Science Press; St. Louis: Missouri Botanical Garden Press.

Csuk, R., Barthel-Niesen, N., Ströhl, D., Kluge, R., Wagner, C. \& AlHarrasi, A. (2015). Tetrahedron, 71, 2025-2034.

Flack, H. D. (1983). Acta Cryst. A39, 876-881.

Hirshfeld, H. L. (1977). Theor. Chim. Acta, 44, 129-138.

Kuete, V., Sandjo, L. P., Mbaveng, A. T., Seukep, J. A., Ngadjui, B. T. \& Efferth, T. (2015). BMC Complementary Alternative Med. 309, 19.

Mesia, K., Tona, L., Mampunza, M. M., Ntamabyaliro, N., Muanda, T., Muyembe, T., Cimanga, K., Totté, J., Mets, T., Pieters, L. \& Vlietinck, A. J. (2012). Planta Med. 78, 211-218.

Sheldrick, G. M. (2008). Acta Cryst. A64, 112-122.

Spackman, M. A. \& Jayatilaka, D. (2009). CrystEngComm, 11, 19 32.

Spek, A. L. (2009). Acta Cryst. D65, 148-155.

Westrip, S. P. (2010). J. Appl. Cryst. 43, 920-925.

Wolff, S. K., Grimwood, D. J., McKinnon, J. J., Turner, M. J., Jayatilaka, D. \& Spackman, M. A. (2012). Crystal Explorer. University of Western Australia.

Xu, Y.-J., Foubert, K., Dhooghe, L., Lemière, F., Cimanga, K., Mesia, K., Apers, S. \& Pieters, L. (2012). Phytochemistry Lett. 5, 316319.

Zeches, M., Richard, B., Gueye-M'Bahia, L., Le Men-Olivier, L. \& Delaude, C. (1985). J. Nat. Prod. 48, 42-46. 


\section{supporting information}

Acta Cryst. (2017). E73, 763-766 [https://doi.org/10.1107/S2056989017006077]

Crystal structure and Hirshfeld surface analysis of 3-oxours-12-ene-27a,28dioic acid (quafrinoic acid)

Jean Jules Bankeu Kezetas, Stéphanie Dietagoum Madjouka, Rajesh Kumar, Muhammad Shaiq Ali, Bruno Lenta Njakou and Sammer Yousuf

Computing details

Data collection: SMART (Bruker, 2009); cell refinement: SAINT (Bruker, 2009); data reduction: SAINT (Bruker, 2009); program(s) used to solve structure: SHELXTL (Sheldrick, 2008); program(s) used to refine structure: SHELXTL (Sheldrick, 2008); molecular graphics: SHELXTL (Sheldrick, 2008); software used to prepare material for publication: SHELXTL (Sheldrick, 2008), PLATON (Spek, 2009) and publCIF (Westrip, 2010).

3-Oxours-12-ene-27a,28-dioic acid

Crystal data

$\mathrm{C}_{30} \mathrm{H}_{44} \mathrm{O}_{5}$

$M_{r}=484.65$

Monoclinic, $P 2_{1}$

$a=8.3465(2) \AA$

$b=10.9783(3) \AA$

$c=14.6583(4) \AA$

$\beta=101.056(1)^{\circ}$

$V=1318.22(6) \AA^{3}$

$Z=2$

\section{Data collection}

Bruker SMART APEX CCD area-detector diffractometer

Radiation source: fine-focus sealed tube

Graphite monochromator

$\omega$ scans

Absorption correction: multi-scan

(SADABS; Bruker, 2009)

$T_{\min }=0.760, T_{\max }=0.927$

\section{Refinement}

Refinement on $F^{2}$

Least-squares matrix: full

$R\left[F^{2}>2 \sigma\left(F^{2}\right)\right]=0.041$

$w R\left(F^{2}\right)=0.115$

$S=1.06$

5116 reflections

325 parameters

2 restraints
$F(000)=528$

$D_{\mathrm{x}}=1.221 \mathrm{Mg} \mathrm{m}^{-3}$

$\mathrm{Cu} K \alpha$ radiation, $\lambda=1.54178 \AA$

Cell parameters from 9925 reflections

$\theta=5.1-72.1^{\circ}$

$\mu=0.64 \mathrm{~mm}^{-1}$

$T=100 \mathrm{~K}$

Block, colourless

$0.45 \times 0.23 \times 0.12 \mathrm{~mm}$

28696 measured reflections

5116 independent reflections

5023 reflections with $I>2 \sigma(I)$

$R_{\text {int }}=0.042$

$\theta_{\max }=72.2^{\circ}, \theta_{\min }=3.1^{\circ}$

$h=-10 \rightarrow 10$

$k=-12 \rightarrow 13$

$l=-18 \rightarrow 18$

Primary atom site location: structure-invariant direct methods

Secondary atom site location: difference Fourier map

Hydrogen site location: inferred from neighbouring sites

$\mathrm{H}$ atoms treated by a mixture of independent and constrained refinement 
$w=1 /\left[\sigma^{2}\left(F_{\mathrm{o}}^{2}\right)+(0.0718 P)^{2}+0.4209 P\right]$

where $P=\left(F_{\mathrm{o}}^{2}+2 F_{\mathrm{c}}^{2}\right) / 3$

$(\Delta / \sigma)_{\max }<0.001$

$\Delta \rho_{\max }=0.70 \mathrm{e} \AA^{-3}$

$\Delta \rho_{\min }=-0.31$ e $\AA^{-3}$
Extinction correction: SHELXTL (Sheldrick, 2008), $\mathrm{Fc}^{*}=\mathrm{kFc}\left[1+0.001 \mathrm{xFc}^{2} \lambda^{3} / \sin (2 \theta)\right]^{-1 / 4}$

Extinction coefficient: 0.0047 (12)

Absolute structure: Flack, 1983

Absolute structure parameter: 0.14 (17)

Special details

Geometry. All esds (except the esd in the dihedral angle between two 1.s. planes) are estimated using the full covariance matrix. The cell esds are taken into account individually in the estimation of esds in distances, angles and torsion angles; correlations between esds in cell parameters are only used when they are defined by crystal symmetry. An approximate (isotropic) treatment of cell esds is used for estimating esds involving l.s. planes.

Refinement. Refinement of $\mathrm{F}^{2}$ against ALL reflections. The weighted R-factor wR and goodness of fit $\mathrm{S}$ are based on $\mathrm{F}^{2}$, conventional R-factors $R$ are based on $F$, with $F$ set to zero for negative $F^{2}$. The threshold expression of $\mathrm{F}^{2}>2 \operatorname{sigma}\left(\mathrm{F}^{2}\right)$ is used only for calculating R-factors(gt) etc. and is not relevant to the choice of reflections for refinement. R-factors based on $\mathrm{F}^{2}$ are statistically about twice as large as those based on F, and R- factors based on ALL data will be even larger.

Fractional atomic coordinates and isotropic or equivalent isotropic displacement parameters $\left(\AA^{2}\right)$

\begin{tabular}{|c|c|c|c|c|}
\hline & $x$ & $y$ & $z$ & $U_{\text {iso }} * / U_{\text {eq }}$ \\
\hline $\mathrm{O} 1$ & $0.27554(19)$ & $0.9227(2)$ & $0.81577(14)$ & $0.0587(6)$ \\
\hline $\mathrm{O} 2$ & $0.63334(15)$ & $0.29733(12)$ & $0.81088(9)$ & $0.0213(3)$ \\
\hline $\mathrm{O} 3$ & $0.56617(14)$ & $0.43709(13)$ & $0.70046(8)$ & $0.0232(3)$ \\
\hline $\mathrm{O} 4$ & $1.26493(17)$ & $0.36149(16)$ & $0.66220(11)$ & $0.0376(4)$ \\
\hline $\mathrm{O} 5$ & $1.31424(15)$ & $0.25278(16)$ & $0.79245(10)$ & $0.0328(3)$ \\
\hline $\mathrm{C} 1$ & $0.6083(2)$ & $0.84362(18)$ & $0.72071(13)$ & $0.0250(4)$ \\
\hline $\mathrm{H} 1 \mathrm{~A}$ & 0.6715 & 0.8743 & 0.6748 & $0.030 *$ \\
\hline H1B & 0.5291 & 0.7829 & 0.6889 & $0.030^{*}$ \\
\hline $\mathrm{C} 2$ & $0.5145(2)$ & $0.9494(2)$ & $0.75258(15)$ & $0.0315(4)$ \\
\hline $\mathrm{H} 2 \mathrm{~A}$ & 0.5919 & 1.0146 & 0.7786 & $0.038 *$ \\
\hline $\mathrm{H} 2 \mathrm{~B}$ & 0.4378 & 0.9833 & 0.6986 & $0.038 *$ \\
\hline $\mathrm{C} 3$ & $0.4206(2)$ & $0.90781(18)$ & $0.82544(14)$ & $0.0295(4)$ \\
\hline $\mathrm{C} 4$ & $0.5182(2)$ & $0.84228(17)$ & $0.91042(13)$ & $0.0235(4)$ \\
\hline $\mathrm{C} 5$ & 0.62587 (19) & $0.74263(16)$ & $0.87525(12)$ & $0.0190(3)$ \\
\hline $\mathrm{H} 5 \mathrm{~A}$ & 0.5453 & 0.6818 & 0.8430 & $0.023^{*}$ \\
\hline C6 & $0.7330(2)$ & $0.66974(17)$ & $0.95315(12)$ & $0.0217(4)$ \\
\hline H6A & 0.8352 & 0.7148 & 0.9770 & $0.026^{*}$ \\
\hline H6B & 0.6745 & 0.6572 & 1.0051 & $0.026^{*}$ \\
\hline $\mathrm{C} 7$ & $0.7722(2)$ & $0.54683(17)$ & $0.91361(11)$ & $0.0206(3)$ \\
\hline H7A & 0.6692 & 0.5010 & 0.8938 & $0.025^{*}$ \\
\hline H7B & 0.8419 & 0.4994 & 0.9634 & $0.025 *$ \\
\hline $\mathrm{C} 8$ & $0.85976(19)$ & $0.55753(17)$ & $0.83033(11)$ & $0.0186(3)$ \\
\hline C9 & $0.7771(2)$ & $0.65618(17)$ & $0.75961(11)$ & $0.0192(3)$ \\
\hline H9A & 0.6722 & 0.6185 & 0.7277 & $0.023 *$ \\
\hline C10 & $0.7266(2)$ & 0.77963 (16) & $0.80027(12)$ & $0.0204(4)$ \\
\hline C11 & $0.8786(2)$ & $0.67108(18)$ & $0.68322(14)$ & $0.0281(4)$ \\
\hline H11A & 0.8169 & 0.7212 & 0.6321 & $0.034^{*}$ \\
\hline H11B & 0.9813 & 0.7144 & 0.7090 & $0.034 *$ \\
\hline C12 & $0.9178(2)$ & $0.55000(19)$ & $0.64540(13)$ & $0.0262(4)$ \\
\hline $\mathrm{H} 12 \mathrm{~A}$ & 0.9584 & 0.5508 & 0.5891 & $0.031 *$ \\
\hline
\end{tabular}




\begin{tabular}{|c|c|c|c|c|}
\hline $\mathrm{C} 13$ & $0.90136(19)$ & $0.44199(17)$ & $0.68303(11)$ & $0.0195(3)$ \\
\hline $\mathrm{C} 14$ & $0.84932(18)$ & $0.42886(17)$ & $0.77766(11)$ & $0.0175(3)$ \\
\hline $\mathrm{C} 15$ & 0.96004 (19) & $0.33334(16)$ & $0.83675(11)$ & $0.0171(3)$ \\
\hline $\mathrm{H} 15 \mathrm{~A}$ & 1.0692 & 0.3697 & 0.8584 & $0.021 *$ \\
\hline H15B & 0.9137 & 0.3141 & 0.8924 & $0.021^{*}$ \\
\hline $\mathrm{C} 16$ & $0.98026(19)$ & $0.21453(16)$ & $0.78546(12)$ & $0.0185(3)$ \\
\hline $\mathrm{H} 16 \mathrm{~A}$ & 0.8734 & 0.1728 & 0.7695 & $0.022 *$ \\
\hline H16B & 1.0563 & 0.1601 & 0.8269 & $0.022 *$ \\
\hline $\mathrm{C} 17$ & $1.0464(2)$ & $0.23782(16)$ & $0.69610(12)$ & $0.0194(4)$ \\
\hline $\mathrm{C} 18$ & $0.9318(2)$ & $0.32589(17)$ & $0.63186(12)$ & $0.0211(4)$ \\
\hline H18A & 0.9905 & 0.3507 & 0.5814 & $0.025^{*}$ \\
\hline $\mathrm{C} 19$ & $0.6708(2)$ & $0.38454(17)$ & $0.76152(11)$ & $0.0174(3)$ \\
\hline $\mathrm{C} 20$ & $1.0395(2)$ & $0.59073(18)$ & $0.86730(14)$ & $0.0258(4)$ \\
\hline $\mathrm{H} 20 \mathrm{~A}$ & 1.0453 & 0.6693 & 0.8996 & $0.039^{*}$ \\
\hline $\mathrm{H} 20 \mathrm{~B}$ & 1.0973 & 0.5964 & 0.8152 & $0.039^{*}$ \\
\hline $\mathrm{H} 20 \mathrm{C}$ & 1.0904 & 0.5277 & 0.9107 & $0.039^{*}$ \\
\hline $\mathrm{C} 21$ & $0.8701(2)$ & $0.86653(17)$ & $0.83604(14)$ & $0.0254(4)$ \\
\hline $\mathrm{H} 21 \mathrm{~A}$ & 0.9272 & 0.8856 & 0.7853 & $0.038^{*}$ \\
\hline $\mathrm{H} 21 \mathrm{~B}$ & 0.9460 & 0.8273 & 0.8869 & $0.038^{*}$ \\
\hline $\mathrm{H} 21 \mathrm{C}$ & 0.8283 & 0.9419 & 0.8587 & $0.038^{*}$ \\
\hline $\mathrm{C} 22$ & $0.6152(2)$ & $0.93770(19)$ & $0.97522(14)$ & $0.0301(4)$ \\
\hline $\mathrm{H} 22 \mathrm{~A}$ & 0.5396 & 0.9967 & 0.9940 & $0.045^{*}$ \\
\hline $\mathrm{H} 22 \mathrm{~B}$ & 0.6908 & 0.9801 & 0.9425 & $0.045^{*}$ \\
\hline $\mathrm{H} 22 \mathrm{C}$ & 0.6770 & 0.8972 & 1.0305 & $0.045^{*}$ \\
\hline $\mathrm{C} 23$ & $0.3981(2)$ & 0.78061 (19) & $0.96218(14)$ & $0.0283(4)$ \\
\hline $\mathrm{H} 23 \mathrm{~A}$ & 0.3303 & 0.8426 & 0.9844 & $0.042^{*}$ \\
\hline $\mathrm{H} 23 \mathrm{~B}$ & 0.4587 & 0.7351 & 1.0153 & $0.042^{*}$ \\
\hline $\mathrm{H} 23 \mathrm{C}$ & 0.3284 & 0.7246 & 0.9201 & $0.042^{*}$ \\
\hline $\mathrm{C} 24$ & $1.2189(2)$ & $0.29000(17)$ & $0.71987(12)$ & $0.0206(3)$ \\
\hline $\mathrm{C} 25$ & $0.7705(2)$ & 0.26347 (19) & $0.58354(13)$ & $0.0248(4)$ \\
\hline $\mathrm{H} 25 \mathrm{~A}$ & 0.7031 & 0.2461 & 0.6316 & $0.030^{*}$ \\
\hline $\mathrm{C} 26$ & $0.8008(3)$ & $0.1421(2)$ & $0.53560(14)$ & $0.0326(5)$ \\
\hline H26A & 0.8574 & 0.1613 & 0.4831 & $0.039 *$ \\
\hline $\mathrm{C} 27$ & $0.9097(2)$ & $0.0580(2)$ & $0.60201(14)$ & $0.0307(4)$ \\
\hline $\mathrm{H} 27 \mathrm{~A}$ & 0.9316 & -0.0164 & 0.5682 & $0.037^{*}$ \\
\hline H27B & 0.8519 & 0.0331 & 0.6520 & $0.037 *$ \\
\hline $\mathrm{C} 28$ & $1.0706(2)$ & $0.11713(18)$ & $0.64521(14)$ & $0.0266(4)$ \\
\hline $\mathrm{H} 28 \mathrm{~A}$ & 1.1338 & 0.1340 & 0.5959 & $0.032 *$ \\
\hline $\mathrm{H} 28 \mathrm{~B}$ & 1.1348 & 0.0598 & 0.6900 & $0.032 *$ \\
\hline $\mathrm{C} 29$ & $0.6414(3)$ & $0.0764(3)$ & $0.49574(18)$ & $0.0475(6)$ \\
\hline H29A & 0.6656 & 0.0004 & 0.4659 & $0.071^{*}$ \\
\hline $\mathrm{H} 29 \mathrm{~B}$ & 0.5734 & 0.1287 & 0.4497 & $0.071^{*}$ \\
\hline $\mathrm{H} 29 \mathrm{C}$ & 0.5830 & 0.0578 & 0.5460 & $0.071^{*}$ \\
\hline $\mathrm{C} 30$ & $0.6761(3)$ & $0.3486(2)$ & $0.51214(15)$ & $0.0378(5)$ \\
\hline $\mathrm{H} 30 \mathrm{~A}$ & 0.6568 & 0.4257 & 0.5420 & $0.057^{*}$ \\
\hline H30B & 0.5712 & 0.3114 & 0.4847 & $0.057^{*}$ \\
\hline $\mathrm{H} 30 \mathrm{C}$ & 0.7389 & 0.3642 & 0.4633 & $0.057^{*}$ \\
\hline H19A & $0.539(5)$ & $0.281(4)$ & $0.796(3)$ & $0.082(12)^{*}$ \\
\hline
\end{tabular}


Atomic displacement parameters $\left(\AA^{2}\right)$

\begin{tabular}{|c|c|c|c|c|c|c|}
\hline & $U^{11}$ & $U^{22}$ & $U^{33}$ & $U^{12}$ & $U^{13}$ & $U^{23}$ \\
\hline $\mathrm{O} 1$ & $0.0238(8)$ & $0.0760(14)$ & $0.0769(12)$ & $0.0147(8)$ & $0.0113(8)$ & $0.0335(11)$ \\
\hline $\mathrm{O} 2$ & $0.0138(5)$ & $0.0251(7)$ & $0.0263(6)$ & -0.0035 & $0.0067(4)$ & $0.0021(5)$ \\
\hline $\mathrm{O} 3$ & $0.0123(5)$ & $0.0291(7)$ & $0.0266(6)$ & -0.0014 & -0.0003 & $0.0041(5)$ \\
\hline $\mathrm{O} 4$ & $0.0185(6)$ & $0.0464(10)$ & $0.0495(9)$ & $-0.0092(6)$ & $0.0106(6)$ & $0.0124(7)$ \\
\hline O5 & $0.0139(6)$ & $0.0527(9)$ & $0.0318(7)$ & $-0.0003(6)$ & $0.0041(5)$ & $0.0018(6)$ \\
\hline $\mathrm{C} 1$ & $0.0204(8)$ & $0.0245(9)$ & $0.0283(9)$ & $-0.0002(7)$ & $0.0004(7)$ & $0.0056(7)$ \\
\hline $\mathrm{C} 2$ & $0.0255(9)$ & $0.0282(11)$ & $0.0386(10)$ & $0.0046(8)$ & $0.0002(8)$ & $0.0076(8)$ \\
\hline $\mathrm{C} 3$ & $0.0225(9)$ & $0.0230(10)$ & $0.0414(10)$ & $0.0048(7)$ & $0.0024(8)$ & $0.0016(8)$ \\
\hline $\mathrm{C} 4$ & $0.0178(8)$ & $0.0217(9)$ & $0.0303(9)$ & $0.0001(7)$ & $0.0030(7)$ & $-0.0035(7)$ \\
\hline $\mathrm{C} 5$ & $0.0146(7)$ & $0.0187(8)$ & $0.0229(8)$ & $-0.0023(6)$ & $0.0019(6)$ & $-0.0014(6)$ \\
\hline C6 & $0.0226(8)$ & $0.0214(9)$ & $0.0206(8)$ & $-0.0003(7)$ & $0.0029(7)$ & $-0.0018(7)$ \\
\hline $\mathrm{C} 7$ & $0.0209(8)$ & $0.0211(9)$ & $0.0191(7)$ & $-0.0016(7)$ & $0.0026(6)$ & $0.0017(7)$ \\
\hline $\mathrm{C} 8$ & $0.0143(7)$ & $0.0198(8)$ & $0.0212(8)$ & $-0.0002(6)$ & $0.0020(6)$ & $0.0025(7)$ \\
\hline C9 & $0.0162(7)$ & $0.0204(9)$ & $0.0210(8)$ & $-0.0015(6)$ & $0.0034(6)$ & $0.0041(7)$ \\
\hline $\mathrm{C} 10$ & $0.0163(7)$ & $0.0171(9)$ & $0.0273(8)$ & $-0.0006(6)$ & $0.0026(6)$ & $0.0029(7)$ \\
\hline C11 & $0.0308(9)$ & $0.0240(10)$ & $0.0328(10)$ & $0.0003(8)$ & $0.0141(8)$ & $0.0095(7)$ \\
\hline $\mathrm{C} 12$ & $0.0259(9)$ & $0.0295(10)$ & $0.0265(9)$ & $0.0002(8)$ & $0.0137(7)$ & $0.0041(8)$ \\
\hline $\mathrm{C} 13$ & $0.0124(7)$ & $0.0265(9)$ & $0.0199(8)$ & $-0.0019(7)$ & $0.0042(6)$ & $0.0025(7)$ \\
\hline $\mathrm{C} 14$ & $0.0103(7)$ & $0.0217(8)$ & $0.0202(7)$ & $-0.0022(6)$ & $0.0021(5)$ & $0.0028(7)$ \\
\hline $\mathrm{C} 15$ & $0.0118(7)$ & $0.0209(8)$ & $0.0186(7)$ & $-0.0014(6)$ & $0.0028(6)$ & $0.0021(6)$ \\
\hline $\mathrm{C} 16$ & $0.0134(7)$ & $0.0200(9)$ & $0.0226(8)$ & $-0.0007(6)$ & $0.0043(6)$ & $0.0025(7)$ \\
\hline $\mathrm{C} 17$ & $0.0136(7)$ & $0.0224(9)$ & $0.0231(8)$ & $-0.0024(6)$ & $0.0057(6)$ & $-0.0021(7)$ \\
\hline $\mathrm{C} 18$ & $0.0157(7)$ & $0.0294(10)$ & $0.0192(8)$ & $-0.0032(7)$ & $0.0058(6)$ & $0.0013(7)$ \\
\hline C19 & $0.0123(7)$ & $0.0225(9)$ & $0.0186(7)$ & $-0.0009(6)$ & $0.0059(6)$ & $0.0000(6)$ \\
\hline $\mathrm{C} 20$ & $0.0160(8)$ & $0.0222(9)$ & $0.0369(10)$ & $-0.0025(6)$ & $-0.0006(7)$ & $-0.0020(7)$ \\
\hline $\mathrm{C} 21$ & $0.0189(8)$ & $0.0196(9)$ & $0.0371(10)$ & $-0.0039(7)$ & $0.0040(7)$ & $0.0023(7)$ \\
\hline $\mathrm{C} 22$ & $0.0266(9)$ & $0.0245(10)$ & $0.0382(10)$ & $-0.0022(8)$ & $0.0036(8)$ & $-0.0081(8)$ \\
\hline $\mathrm{C} 23$ & $0.0249(9)$ & $0.0262(10)$ & $0.0355(10)$ & $-0.0009(8)$ & $0.0101(7)$ & $-0.0057(8)$ \\
\hline $\mathrm{C} 24$ & $0.0156(7)$ & $0.0243(9)$ & $0.0237(8)$ & $-0.0002(7)$ & $0.0082(6)$ & $-0.0025(7)$ \\
\hline $\mathrm{C} 25$ & $0.0173(8)$ & $0.0331(10)$ & $0.0228(8)$ & $-0.0050(7)$ & $0.0010(6)$ & $-0.0005(7)$ \\
\hline $\mathrm{C} 26$ & $0.0323(10)$ & $0.0416(12)$ & $0.0255(9)$ & $-0.0099(9)$ & $0.0094(8)$ & $-0.0086(9)$ \\
\hline $\mathrm{C} 27$ & $0.0333(10)$ & $0.0301(11)$ & $0.0316(10)$ & $-0.0093(9)$ & $0.0137(8)$ & $-0.0097(8)$ \\
\hline $\mathrm{C} 28$ & $0.0243(9)$ & $0.0261(10)$ & $0.0317(10)$ & $-0.0007(7)$ & $0.0109(7)$ & $-0.0041(8)$ \\
\hline $\mathrm{C} 29$ & $0.0463(13)$ & $0.0494(15)$ & $0.0411(12)$ & $-0.0124(12)$ & $-0.0062(10)$ & $-0.0105(11)$ \\
\hline C30 & $0.0314(10)$ & 0.0439 & $0.0355(11)$ & $0.0079(9)$ & $0.0003(8)$ & $-0.0029(9)$ \\
\hline
\end{tabular}

Geometric parameters $\left(\AA,{ }^{\circ}\right)$

\begin{tabular}{llll}
\hline $\mathrm{O} 1-\mathrm{C} 3$ & $1.203(3)$ & $\mathrm{C} 14-\mathrm{C} 15$ & $1.548(2)$ \\
$\mathrm{O} 2-\mathrm{C} 19$ & $1.275(2)$ & $\mathrm{C} 15-\mathrm{C} 16$ & $1.531(2)$ \\
$\mathrm{O} 2-\mathrm{H} 19 \mathrm{~A}$ & $0.79(4)$ & $\mathrm{C} 15-\mathrm{H} 15 \mathrm{~A}$ & 0.9900 \\
$\mathrm{O} 3-\mathrm{C} 19$ & $1.264(2)$ & $\mathrm{C} 15-\mathrm{H} 15 \mathrm{~B}$ & 0.9900 \\
$\mathrm{O} 4-\mathrm{C} 24$ & $1.266(2)$ & $\mathrm{C} 16-\mathrm{C} 17$ & $1.537(2)$
\end{tabular}




\begin{tabular}{|c|c|c|c|}
\hline $\mathrm{O} 4-\mathrm{H} 4 \mathrm{~A}$ & $0.906(10)$ & C16-H16A & 0.9900 \\
\hline $\mathrm{O} 5-\mathrm{C} 24$ & $1.268(2)$ & C16-H16B & 0.9900 \\
\hline $\mathrm{C} 1-\mathrm{C} 2$ & $1.523(3)$ & $\mathrm{C} 17-\mathrm{C} 24$ & $1.526(2)$ \\
\hline $\mathrm{C} 1-\mathrm{C} 10$ & $1.545(2)$ & $\mathrm{C} 17-\mathrm{C} 18$ & $1.546(2)$ \\
\hline $\mathrm{C} 1-\mathrm{H} 1 \mathrm{~A}$ & 0.9900 & $\mathrm{C} 17-\mathrm{C} 28$ & $1.553(3)$ \\
\hline $\mathrm{C} 1-\mathrm{H} 1 \mathrm{~B}$ & 0.9900 & $\mathrm{C} 18-\mathrm{C} 25$ & $1.555(2)$ \\
\hline $\mathrm{C} 2-\mathrm{C} 3$ & $1.511(3)$ & $\mathrm{C} 18-\mathrm{H} 18 \mathrm{~A}$ & 1.0000 \\
\hline $\mathrm{C} 2-\mathrm{H} 2 \mathrm{~A}$ & 0.9900 & $\mathrm{C} 20-\mathrm{H} 20 \mathrm{~A}$ & 0.9800 \\
\hline $\mathrm{C} 2-\mathrm{H} 2 \mathrm{~B}$ & 0.9900 & $\mathrm{C} 20-\mathrm{H} 20 \mathrm{~B}$ & 0.9800 \\
\hline $\mathrm{C} 3-\mathrm{C} 4$ & $1.531(3)$ & $\mathrm{C} 20-\mathrm{H} 20 \mathrm{C}$ & 0.9800 \\
\hline $\mathrm{C} 4-\mathrm{C} 23$ & $1.526(3)$ & $\mathrm{C} 21-\mathrm{H} 21 \mathrm{~A}$ & 0.9800 \\
\hline $\mathrm{C} 4-\mathrm{C} 22$ & $1.536(3)$ & $\mathrm{C} 21-\mathrm{H} 21 \mathrm{~B}$ & 0.9800 \\
\hline $\mathrm{C} 4-\mathrm{C} 5$ & $1.565(2)$ & $\mathrm{C} 21-\mathrm{H} 21 \mathrm{C}$ & 0.9800 \\
\hline $\mathrm{C} 5-\mathrm{C} 6$ & $1.534(2)$ & $\mathrm{C} 22-\mathrm{H} 22 \mathrm{~A}$ & 0.9800 \\
\hline $\mathrm{C} 5-\mathrm{C} 10$ & $1.560(2)$ & $\mathrm{C} 22-\mathrm{H} 22 \mathrm{~B}$ & 0.9800 \\
\hline $\mathrm{C} 5-\mathrm{H} 5 \mathrm{~A}$ & 1.0000 & $\mathrm{C} 22-\mathrm{H} 22 \mathrm{C}$ & 0.9800 \\
\hline $\mathrm{C} 6-\mathrm{C} 7$ & $1.529(2)$ & $\mathrm{C} 23-\mathrm{H} 23 \mathrm{~A}$ & 0.9800 \\
\hline C6-H6A & 0.9900 & $\mathrm{C} 23-\mathrm{H} 23 \mathrm{~B}$ & 0.9800 \\
\hline $\mathrm{C} 6-\mathrm{H} 6 \mathrm{~B}$ & 0.9900 & $\mathrm{C} 23-\mathrm{H} 23 \mathrm{C}$ & 0.9800 \\
\hline $\mathrm{C} 7-\mathrm{C} 8$ & $1.543(2)$ & $\mathrm{C} 25-\mathrm{C} 30$ & $1.508(3)$ \\
\hline C7-H7A & 0.9900 & $\mathrm{C} 25-\mathrm{C} 26$ & $1.550(3)$ \\
\hline C7-H7B & 0.9900 & $\mathrm{C} 25-\mathrm{H} 25 \mathrm{~A}$ & 1.0000 \\
\hline $\mathrm{C} 8-\mathrm{C} 20$ & $1.538(2)$ & $\mathrm{C} 26-\mathrm{C} 27$ & $1.512(3)$ \\
\hline $\mathrm{C} 8-\mathrm{C} 9$ & $1.565(2)$ & $\mathrm{C} 26-\mathrm{C} 29$ & $1.528(3)$ \\
\hline $\mathrm{C} 8-\mathrm{C} 14$ & $1.604(2)$ & $\mathrm{C} 26-\mathrm{H} 26 \mathrm{~A}$ & 1.0000 \\
\hline $\mathrm{C} 9-\mathrm{C} 11$ & $1.537(2)$ & $\mathrm{C} 27-\mathrm{C} 28$ & $1.517(3)$ \\
\hline $\mathrm{C} 9-\mathrm{C} 10$ & $1.571(2)$ & $\mathrm{C} 27-\mathrm{H} 27 \mathrm{~A}$ & 0.9900 \\
\hline C9-H9A & 1.0000 & $\mathrm{C} 27-\mathrm{H} 27 \mathrm{~B}$ & 0.9900 \\
\hline $\mathrm{C} 10-\mathrm{C} 21$ & $1.543(2)$ & $\mathrm{C} 28-\mathrm{H} 28 \mathrm{~A}$ & 0.9900 \\
\hline $\mathrm{C} 11-\mathrm{C} 12$ & $1.501(3)$ & $\mathrm{C} 28-\mathrm{H} 28 \mathrm{~B}$ & 0.9900 \\
\hline $\mathrm{C} 11-\mathrm{H} 11 \mathrm{~A}$ & 0.9900 & $\mathrm{C} 29-\mathrm{H} 29 \mathrm{~A}$ & 0.9800 \\
\hline $\mathrm{C} 11-\mathrm{H} 11 \mathrm{~B}$ & 0.9900 & $\mathrm{C} 29-\mathrm{H} 29 \mathrm{~B}$ & 0.9800 \\
\hline $\mathrm{C} 12-\mathrm{C} 13$ & $1.326(3)$ & $\mathrm{C} 29-\mathrm{H} 29 \mathrm{C}$ & 0.9800 \\
\hline $\mathrm{C} 12-\mathrm{H} 12 \mathrm{~A}$ & 0.9500 & $\mathrm{C} 30-\mathrm{H} 30 \mathrm{~A}$ & 0.9800 \\
\hline $\mathrm{C} 13-\mathrm{C} 18$ & $1.525(3)$ & $\mathrm{C} 30-\mathrm{H} 30 \mathrm{~B}$ & 0.9800 \\
\hline $\mathrm{C} 13-\mathrm{C} 14$ & $1.538(2)$ & $\mathrm{C} 30-\mathrm{H} 30 \mathrm{C}$ & 0.9800 \\
\hline $\mathrm{C} 14-\mathrm{C} 19$ & $1.542(2)$ & & \\
\hline $\mathrm{C} 19-\mathrm{O} 2-\mathrm{H} 19 \mathrm{~A}$ & $111(3)$ & $\mathrm{C} 15-\mathrm{C} 16-\mathrm{H} 16 \mathrm{~A}$ & 109.3 \\
\hline $\mathrm{C} 24-\mathrm{O} 4-\mathrm{H} 4 \mathrm{~A}$ & $115(3)$ & $\mathrm{C} 17-\mathrm{C} 16-\mathrm{H} 16 \mathrm{~A}$ & 109.3 \\
\hline $\mathrm{C} 2-\mathrm{C} 1-\mathrm{C} 10$ & $113.99(16)$ & $\mathrm{C} 15-\mathrm{C} 16-\mathrm{H} 16 \mathrm{~B}$ & 109.3 \\
\hline $\mathrm{C} 2-\mathrm{C} 1-\mathrm{H} 1 \mathrm{~A}$ & 108.8 & $\mathrm{C} 17-\mathrm{C} 16-\mathrm{H} 16 \mathrm{~B}$ & 109.3 \\
\hline $\mathrm{C} 10-\mathrm{C} 1-\mathrm{H} 1 \mathrm{~A}$ & 108.8 & $\mathrm{H} 16 \mathrm{~A}-\mathrm{C} 16-\mathrm{H} 16 \mathrm{~B}$ & 108.0 \\
\hline $\mathrm{C} 2-\mathrm{C} 1-\mathrm{H} 1 \mathrm{~B}$ & 108.8 & $\mathrm{C} 24-\mathrm{C} 17-\mathrm{C} 16$ & $110.18(13)$ \\
\hline $\mathrm{C} 10-\mathrm{C} 1-\mathrm{H} 1 \mathrm{~B}$ & 108.8 & $\mathrm{C} 24-\mathrm{C} 17-\mathrm{C} 18$ & $110.52(14)$ \\
\hline $\mathrm{H} 1 \mathrm{~A}-\mathrm{C} 1-\mathrm{H} 1 \mathrm{~B}$ & 107.6 & $\mathrm{C} 16-\mathrm{C} 17-\mathrm{C} 18$ & $109.97(13)$ \\
\hline $\mathrm{C} 3-\mathrm{C} 2-\mathrm{C} 1$ & $110.69(17)$ & $\mathrm{C} 24-\mathrm{C} 17-\mathrm{C} 28$ & $103.06(13)$ \\
\hline $\mathrm{C} 3-\mathrm{C} 2-\mathrm{H} 2 \mathrm{~A}$ & 109.5 & $\mathrm{C} 16-\mathrm{C} 17-\mathrm{C} 28$ & $111.63(15)$ \\
\hline
\end{tabular}




\begin{tabular}{|c|c|c|c|}
\hline $\mathrm{C} 1-\mathrm{C} 2-\mathrm{H} 2 \mathrm{~A}$ & 109.5 & $\mathrm{C} 18-\mathrm{C} 17-\mathrm{C} 28$ & $111.31(14)$ \\
\hline $\mathrm{C} 3-\mathrm{C} 2-\mathrm{H} 2 \mathrm{~B}$ & 109.5 & $\mathrm{C} 13-\mathrm{C} 18-\mathrm{C} 17$ & $111.42(13)$ \\
\hline $\mathrm{C} 1-\mathrm{C} 2-\mathrm{H} 2 \mathrm{~B}$ & 109.5 & $\mathrm{C} 13-\mathrm{C} 18-\mathrm{C} 25$ & $112.39(14)$ \\
\hline $\mathrm{H} 2 \mathrm{~A}-\mathrm{C} 2-\mathrm{H} 2 \mathrm{~B}$ & 108.1 & $\mathrm{C} 17-\mathrm{C} 18-\mathrm{C} 25$ & $112.48(15)$ \\
\hline $\mathrm{O} 1-\mathrm{C} 3-\mathrm{C} 2$ & $121.49(18)$ & $\mathrm{C} 13-\mathrm{C} 18-\mathrm{H} 18 \mathrm{~A}$ & 106.7 \\
\hline $\mathrm{O} 1-\mathrm{C} 3-\mathrm{C} 4$ & $121.75(19)$ & $\mathrm{C} 17-\mathrm{C} 18-\mathrm{H} 18 \mathrm{~A}$ & 106.7 \\
\hline $\mathrm{C} 2-\mathrm{C} 3-\mathrm{C} 4$ & $116.73(16)$ & $\mathrm{C} 25-\mathrm{C} 18-\mathrm{H} 18 \mathrm{~A}$ & 106.7 \\
\hline $\mathrm{C} 23-\mathrm{C} 4-\mathrm{C} 3$ & $108.39(15)$ & $\mathrm{O} 3-\mathrm{C} 19-\mathrm{O} 2$ & $122.26(15)$ \\
\hline $\mathrm{C} 23-\mathrm{C} 4-\mathrm{C} 22$ & $108.26(15)$ & $\mathrm{O} 3-\mathrm{C} 19-\mathrm{C} 14$ & $118.73(15)$ \\
\hline $\mathrm{C} 3-\mathrm{C} 4-\mathrm{C} 22$ & $108.53(16)$ & $\mathrm{O} 2-\mathrm{C} 19-\mathrm{C} 14$ & $119.00(15)$ \\
\hline $\mathrm{C} 23-\mathrm{C} 4-\mathrm{C} 5$ & $109.09(15)$ & $\mathrm{C} 8-\mathrm{C} 20-\mathrm{H} 20 \mathrm{~A}$ & 109.5 \\
\hline $\mathrm{C} 3-\mathrm{C} 4-\mathrm{C} 5$ & $108.06(15)$ & $\mathrm{C} 8-\mathrm{C} 20-\mathrm{H} 20 \mathrm{~B}$ & 109.5 \\
\hline $\mathrm{C} 22-\mathrm{C} 4-\mathrm{C} 5$ & $114.37(14)$ & $\mathrm{H} 20 \mathrm{~A}-\mathrm{C} 20-\mathrm{H} 20 \mathrm{~B}$ & 109.5 \\
\hline $\mathrm{C} 6-\mathrm{C} 5-\mathrm{C} 10$ & $110.16(13)$ & $\mathrm{C} 8-\mathrm{C} 20-\mathrm{H} 20 \mathrm{C}$ & 109.5 \\
\hline $\mathrm{C} 6-\mathrm{C} 5-\mathrm{C} 4$ & $114.15(14)$ & $\mathrm{H} 20 \mathrm{~A}-\mathrm{C} 20-\mathrm{H} 20 \mathrm{C}$ & 109.5 \\
\hline $\mathrm{C} 10-\mathrm{C} 5-\mathrm{C} 4$ & $118.11(14)$ & $\mathrm{H} 20 \mathrm{~B}-\mathrm{C} 20-\mathrm{H} 20 \mathrm{C}$ & 109.5 \\
\hline $\mathrm{C} 6-\mathrm{C} 5-\mathrm{H} 5 \mathrm{~A}$ & 104.2 & $\mathrm{C} 10-\mathrm{C} 21-\mathrm{H} 21 \mathrm{~A}$ & 109.5 \\
\hline $\mathrm{C} 10-\mathrm{C} 5-\mathrm{H} 5 \mathrm{~A}$ & 104.2 & $\mathrm{C} 10-\mathrm{C} 21-\mathrm{H} 21 \mathrm{~B}$ & 109.5 \\
\hline $\mathrm{C} 4-\mathrm{C} 5-\mathrm{H} 5 \mathrm{~A}$ & 104.2 & $\mathrm{H} 21 \mathrm{~A}-\mathrm{C} 21-\mathrm{H} 21 \mathrm{~B}$ & 109.5 \\
\hline $\mathrm{C} 7-\mathrm{C} 6-\mathrm{C} 5$ & $108.36(13)$ & $\mathrm{C} 10-\mathrm{C} 21-\mathrm{H} 21 \mathrm{C}$ & 109.5 \\
\hline $\mathrm{C} 7-\mathrm{C} 6-\mathrm{H} 6 \mathrm{~A}$ & 110.0 & $\mathrm{H} 21 \mathrm{~A}-\mathrm{C} 21-\mathrm{H} 21 \mathrm{C}$ & 109.5 \\
\hline $\mathrm{C} 5-\mathrm{C} 6-\mathrm{H} 6 \mathrm{~A}$ & 110.0 & $\mathrm{H} 21 \mathrm{~B}-\mathrm{C} 21-\mathrm{H} 21 \mathrm{C}$ & 109.5 \\
\hline $\mathrm{C} 7-\mathrm{C} 6-\mathrm{H} 6 \mathrm{~B}$ & 110.0 & $\mathrm{C} 4-\mathrm{C} 22-\mathrm{H} 22 \mathrm{~A}$ & 109.5 \\
\hline $\mathrm{C} 5-\mathrm{C} 6-\mathrm{H} 6 \mathrm{~B}$ & 110.0 & $\mathrm{C} 4-\mathrm{C} 22-\mathrm{H} 22 \mathrm{~B}$ & 109.5 \\
\hline $\mathrm{H} 6 \mathrm{~A}-\mathrm{C} 6-\mathrm{H} 6 \mathrm{~B}$ & 108.4 & $\mathrm{H} 22 \mathrm{~A}-\mathrm{C} 22-\mathrm{H} 22 \mathrm{~B}$ & 109.5 \\
\hline $\mathrm{C} 6-\mathrm{C} 7-\mathrm{C} 8$ & $113.65(15)$ & $\mathrm{C} 4-\mathrm{C} 22-\mathrm{H} 22 \mathrm{C}$ & 109.5 \\
\hline $\mathrm{C} 6-\mathrm{C} 7-\mathrm{H} 7 \mathrm{~A}$ & 108.8 & $\mathrm{H} 22 \mathrm{~A}-\mathrm{C} 22-\mathrm{H} 22 \mathrm{C}$ & 109.5 \\
\hline $\mathrm{C} 8-\mathrm{C} 7-\mathrm{H} 7 \mathrm{~A}$ & 108.8 & $\mathrm{H} 22 \mathrm{~B}-\mathrm{C} 22-\mathrm{H} 22 \mathrm{C}$ & 109.5 \\
\hline $\mathrm{C} 6-\mathrm{C} 7-\mathrm{H} 7 \mathrm{~B}$ & 108.8 & $\mathrm{C} 4-\mathrm{C} 23-\mathrm{H} 23 \mathrm{~A}$ & 109.5 \\
\hline $\mathrm{C} 8-\mathrm{C} 7-\mathrm{H} 7 \mathrm{~B}$ & 108.8 & $\mathrm{C} 4-\mathrm{C} 23-\mathrm{H} 23 \mathrm{~B}$ & 109.5 \\
\hline $\mathrm{H} 7 \mathrm{~A}-\mathrm{C} 7-\mathrm{H} 7 \mathrm{~B}$ & 107.7 & $\mathrm{H} 23 \mathrm{~A}-\mathrm{C} 23-\mathrm{H} 23 \mathrm{~B}$ & 109.5 \\
\hline $\mathrm{C} 20-\mathrm{C} 8-\mathrm{C} 7$ & $108.50(14)$ & $\mathrm{C} 4-\mathrm{C} 23-\mathrm{H} 23 \mathrm{C}$ & 109.5 \\
\hline $\mathrm{C} 20-\mathrm{C} 8-\mathrm{C} 9$ & $110.22(14)$ & $\mathrm{H} 23 \mathrm{~A}-\mathrm{C} 23-\mathrm{H} 23 \mathrm{C}$ & 109.5 \\
\hline $\mathrm{C} 7-\mathrm{C} 8-\mathrm{C} 9$ & $111.18(13)$ & $\mathrm{H} 23 \mathrm{~B}-\mathrm{C} 23-\mathrm{H} 23 \mathrm{C}$ & 109.5 \\
\hline $\mathrm{C} 20-\mathrm{C} 8-\mathrm{C} 14$ & $109.73(14)$ & $\mathrm{O} 4-\mathrm{C} 24-\mathrm{O} 5$ & $122.53(16)$ \\
\hline $\mathrm{C} 7-\mathrm{C} 8-\mathrm{C} 14$ & $108.88(14)$ & $\mathrm{O} 4-\mathrm{C} 24-\mathrm{C} 17$ & $118.30(15)$ \\
\hline $\mathrm{C} 9-\mathrm{C} 8-\mathrm{C} 14$ & $108.31(12)$ & $\mathrm{O} 5-\mathrm{C} 24-\mathrm{C} 17$ & $118.91(15)$ \\
\hline $\mathrm{C} 11-\mathrm{C} 9-\mathrm{C} 8$ & $108.79(14)$ & $\mathrm{C} 30-\mathrm{C} 25-\mathrm{C} 26$ & $109.11(16)$ \\
\hline $\mathrm{C} 11-\mathrm{C} 9-\mathrm{C} 10$ & $114.23(15)$ & $\mathrm{C} 30-\mathrm{C} 25-\mathrm{C} 18$ & $109.54(17)$ \\
\hline $\mathrm{C} 8-\mathrm{C} 9-\mathrm{C} 10$ & $117.53(13)$ & $\mathrm{C} 26-\mathrm{C} 25-\mathrm{C} 18$ & $112.50(15)$ \\
\hline $\mathrm{C} 11-\mathrm{C} 9-\mathrm{H} 9 \mathrm{~A}$ & 105.0 & $\mathrm{C} 30-\mathrm{C} 25-\mathrm{H} 25 \mathrm{~A}$ & 108.5 \\
\hline $\mathrm{C} 8-\mathrm{C} 9-\mathrm{H} 9 \mathrm{~A}$ & 105.0 & $\mathrm{C} 26-\mathrm{C} 25-\mathrm{H} 25 \mathrm{~A}$ & 108.5 \\
\hline $\mathrm{C} 10-\mathrm{C} 9-\mathrm{H} 9 \mathrm{~A}$ & 105.0 & $\mathrm{C} 18-\mathrm{C} 25-\mathrm{H} 25 \mathrm{~A}$ & 108.5 \\
\hline $\mathrm{C} 21-\mathrm{C} 10-\mathrm{C} 1$ & $108.51(14)$ & $\mathrm{C} 27-\mathrm{C} 26-\mathrm{C} 29$ & $109.2(2)$ \\
\hline $\mathrm{C} 21-\mathrm{C} 10-\mathrm{C} 5$ & $114.20(14)$ & $\mathrm{C} 27-\mathrm{C} 26-\mathrm{C} 25$ & $111.32(15)$ \\
\hline $\mathrm{C} 1-\mathrm{C} 10-\mathrm{C} 5$ & $107.32(13)$ & $\mathrm{C} 29-\mathrm{C} 26-\mathrm{C} 25$ & $111.93(19)$ \\
\hline $\mathrm{C} 21-\mathrm{C} 10-\mathrm{C} 9$ & $114.51(14)$ & $\mathrm{C} 27-\mathrm{C} 26-\mathrm{H} 26 \mathrm{~A}$ & 108.1 \\
\hline $\mathrm{C} 1-\mathrm{C} 10-\mathrm{C} 9$ & $106.57(14)$ & $\mathrm{C} 29-\mathrm{C} 26-\mathrm{H} 26 \mathrm{~A}$ & 108.1 \\
\hline
\end{tabular}




\begin{tabular}{|c|c|}
\hline $\mathrm{C} 5-\mathrm{C} 10-\mathrm{C} 9$ & $105.25(13)$ \\
\hline $\mathrm{C} 12-\mathrm{C} 11-\mathrm{C} 9$ & $111.40(15)$ \\
\hline $\mathrm{C} 12-\mathrm{C} 11-\mathrm{H} 11 \mathrm{~A}$ & 109.3 \\
\hline C9-C11-H11A & 109.3 \\
\hline $\mathrm{C} 12-\mathrm{C} 11-\mathrm{H} 11 \mathrm{~B}$ & 109.3 \\
\hline $\mathrm{C} 9-\mathrm{C} 11-\mathrm{H} 11 \mathrm{~B}$ & 109.3 \\
\hline $\mathrm{H} 11 \mathrm{~A}-\mathrm{C} 11-\mathrm{H} 11 \mathrm{~B}$ & 108.0 \\
\hline $\mathrm{C} 13-\mathrm{C} 12-\mathrm{C} 11$ & $126.23(15)$ \\
\hline $\mathrm{C} 13-\mathrm{C} 12-\mathrm{H} 12 \mathrm{~A}$ & 116.9 \\
\hline $\mathrm{C} 11-\mathrm{C} 12-\mathrm{H} 12 \mathrm{~A}$ & 116.9 \\
\hline $\mathrm{C} 12-\mathrm{C} 13-\mathrm{C} 18$ & $120.17(15)$ \\
\hline $\mathrm{C} 12-\mathrm{C} 13-\mathrm{C} 14$ & $121.93(17)$ \\
\hline $\mathrm{C} 18-\mathrm{C} 13-\mathrm{C} 14$ & $117.89(15)$ \\
\hline $\mathrm{C} 13-\mathrm{C} 14-\mathrm{C} 19$ & $108.85(13)$ \\
\hline $\mathrm{C} 13-\mathrm{C} 14-\mathrm{C} 15$ & $109.06(13)$ \\
\hline $\mathrm{C} 19-\mathrm{C} 14-\mathrm{C} 15$ & $109.13(14)$ \\
\hline $\mathrm{C} 13-\mathrm{C} 14-\mathrm{C} 8$ & $110.66(14)$ \\
\hline $\mathrm{C} 19-\mathrm{C} 14-\mathrm{C} 8$ & $108.26(13)$ \\
\hline $\mathrm{C} 15-\mathrm{C} 14-\mathrm{C} 8$ & $110.83(12)$ \\
\hline $\mathrm{C} 16-\mathrm{C} 15-\mathrm{C} 14$ & $114.36(13)$ \\
\hline $\mathrm{C} 16-\mathrm{C} 15-\mathrm{H} 15 \mathrm{~A}$ & 108.7 \\
\hline $\mathrm{C} 14-\mathrm{C} 15-\mathrm{H} 15 \mathrm{~A}$ & 108.7 \\
\hline $\mathrm{C} 16-\mathrm{C} 15-\mathrm{H} 15 \mathrm{~B}$ & 108.7 \\
\hline $\mathrm{C} 14-\mathrm{C} 15-\mathrm{H} 15 \mathrm{~B}$ & 108.7 \\
\hline $\mathrm{H} 15 \mathrm{~A}-\mathrm{C} 15-\mathrm{H} 15 \mathrm{~B}$ & 107.6 \\
\hline $\mathrm{C} 15-\mathrm{C} 16-\mathrm{C} 17$ & $111.60(14)$ \\
\hline $\mathrm{C} 10-\mathrm{C} 1-\mathrm{C} 2-\mathrm{C} 3$ & $-56.5(2)$ \\
\hline $\mathrm{C} 1-\mathrm{C} 2-\mathrm{C} 3-\mathrm{O} 1$ & $-124.2(2)$ \\
\hline $\mathrm{C} 1-\mathrm{C} 2-\mathrm{C} 3-\mathrm{C} 4$ & $53.9(2)$ \\
\hline $\mathrm{O} 1-\mathrm{C} 3-\mathrm{C} 4-\mathrm{C} 23$ & $12.1(3)$ \\
\hline $\mathrm{C} 2-\mathrm{C} 3-\mathrm{C} 4-\mathrm{C} 23$ & $-165.95(17)$ \\
\hline $\mathrm{O} 1-\mathrm{C} 3-\mathrm{C} 4-\mathrm{C} 22$ & $-105.2(2)$ \\
\hline $\mathrm{C} 2-\mathrm{C} 3-\mathrm{C} 4-\mathrm{C} 22$ & $76.7(2)$ \\
\hline $\mathrm{O} 1-\mathrm{C} 3-\mathrm{C} 4-\mathrm{C} 5$ & $130.2(2)$ \\
\hline $\mathrm{C} 2-\mathrm{C} 3-\mathrm{C} 4-\mathrm{C} 5$ & $-47.9(2)$ \\
\hline $\mathrm{C} 23-\mathrm{C} 4-\mathrm{C} 5-\mathrm{C} 6$ & $-63.04(19)$ \\
\hline $\mathrm{C} 3-\mathrm{C} 4-\mathrm{C} 5-\mathrm{C} 6$ & $179.32(15)$ \\
\hline $\mathrm{C} 22-\mathrm{C} 4-\mathrm{C} 5-\mathrm{C} 6$ & $58.3(2)$ \\
\hline $\mathrm{C} 23-\mathrm{C} 4-\mathrm{C} 5-\mathrm{C} 10$ & $165.07(15)$ \\
\hline $\mathrm{C} 3-\mathrm{C} 4-\mathrm{C} 5-\mathrm{C} 10$ & $47.43(19)$ \\
\hline $\mathrm{C} 22-\mathrm{C} 4-\mathrm{C} 5-\mathrm{C} 10$ & $-73.5(2)$ \\
\hline $\mathrm{C} 10-\mathrm{C} 5-\mathrm{C} 6-\mathrm{C} 7$ & $-68.31(17)$ \\
\hline $\mathrm{C} 4-\mathrm{C} 5-\mathrm{C} 6-\mathrm{C} 7$ & $156.07(14)$ \\
\hline $\mathrm{C} 5-\mathrm{C} 6-\mathrm{C} 7-\mathrm{C} 8$ & $58.19(18)$ \\
\hline $\mathrm{C} 6-\mathrm{C} 7-\mathrm{C} 8-\mathrm{C} 20$ & $76.36(18)$ \\
\hline $\mathrm{C} 6-\mathrm{C} 7-\mathrm{C} 8-\mathrm{C} 9$ & $-45.00(19)$ \\
\hline $\mathrm{C} 6-\mathrm{C} 7-\mathrm{C} 8-\mathrm{C} 14$ & $-164.25(13)$ \\
\hline
\end{tabular}

$\mathrm{C} 25-\mathrm{C} 26-\mathrm{H} 26 \mathrm{~A} \quad 108.1$

$\mathrm{C} 26-\mathrm{C} 27-\mathrm{C} 28 \quad 112.44$ (17)

$\mathrm{C} 26-\mathrm{C} 27-\mathrm{H} 27 \mathrm{~A} \quad 109.1$

$\mathrm{C} 28-\mathrm{C} 27-\mathrm{H} 27 \mathrm{~A} \quad 109.1$

$\mathrm{C} 26-\mathrm{C} 27-\mathrm{H} 27 \mathrm{~B} \quad 109.1$

$\mathrm{C} 28-\mathrm{C} 27-\mathrm{H} 27 \mathrm{~B} \quad 109.1$

$\mathrm{H} 27 \mathrm{~A}-\mathrm{C} 27-\mathrm{H} 27 \mathrm{~B} \quad 107.8$

$\mathrm{C} 27-\mathrm{C} 28-\mathrm{C} 17 \quad 112.29(15)$

$\mathrm{C} 27-\mathrm{C} 28-\mathrm{H} 28 \mathrm{~A} \quad 109.1$

$\mathrm{C} 17-\mathrm{C} 28-\mathrm{H} 28 \mathrm{~A} \quad 109.1$

$\mathrm{C} 27-\mathrm{C} 28-\mathrm{H} 28 \mathrm{~B} \quad 109.1$

$\mathrm{C} 17-\mathrm{C} 28-\mathrm{H} 28 \mathrm{~B} \quad 109.1$

$\mathrm{H} 28 \mathrm{~A}-\mathrm{C} 28-\mathrm{H} 28 \mathrm{~B} \quad 107.9$

$\mathrm{C} 26-\mathrm{C} 29-\mathrm{H} 29 \mathrm{~A} \quad 109.5$

$\mathrm{C} 26-\mathrm{C} 29-\mathrm{H} 29 \mathrm{~B} \quad 109.5$

$\mathrm{H} 29 \mathrm{~A}-\mathrm{C} 29-\mathrm{H} 29 \mathrm{~B} \quad 109.5$

C26-C29- $\mathrm{H} 29 \mathrm{C} \quad 109.5$

$\mathrm{H} 29 \mathrm{~A}-\mathrm{C} 29-\mathrm{H} 29 \mathrm{C} \quad 109.5$

$\mathrm{H} 29 \mathrm{~B}-\mathrm{C} 29-\mathrm{H} 29 \mathrm{C} \quad 109.5$

$\mathrm{C} 25-\mathrm{C} 30-\mathrm{H} 30 \mathrm{~A} \quad 109.5$

$\mathrm{C} 25-\mathrm{C} 30-\mathrm{H} 30 \mathrm{~B} \quad 109.5$

$\mathrm{H} 30 \mathrm{~A}-\mathrm{C} 30-\mathrm{H} 30 \mathrm{~B} \quad 109.5$

$\mathrm{C} 25-\mathrm{C} 30-\mathrm{H} 30 \mathrm{C} \quad 109.5$

$\mathrm{H} 30 \mathrm{~A}-\mathrm{C} 30-\mathrm{H} 30 \mathrm{C} \quad 109.5$

$\mathrm{H} 30 \mathrm{~B}-\mathrm{C} 30-\mathrm{H} 30 \mathrm{C} \quad 109.5$

$\begin{array}{ll}\mathrm{C} 20-\mathrm{C} 8-\mathrm{C} 14-\mathrm{C} 13 & -73.60(17) \\ \mathrm{C} 7-\mathrm{C} 8-\mathrm{C} 14-\mathrm{C} 13 & 167.79(13) \\ \mathrm{C} 9-\mathrm{C} 8-\mathrm{C} 14-\mathrm{C} 13 & 46.77(16) \\ \mathrm{C} 20-\mathrm{C} 8-\mathrm{C} 14-\mathrm{C} 19 & 167.19(14) \\ \mathrm{C} 7-\mathrm{C} 8-\mathrm{C} 14-\mathrm{C} 19 & 48.58(16) \\ \mathrm{C} 9-\mathrm{C} 8-\mathrm{C} 14-\mathrm{C} 19 & -72.45(15) \\ \mathrm{C} 20-\mathrm{C} 8-\mathrm{C} 14-\mathrm{C} 15 & 47.54(17) \\ \mathrm{C} 7-\mathrm{C} 8-\mathrm{C} 14-\mathrm{C} 15 & -71.08(15) \\ \mathrm{C} 9-\mathrm{C} 8-\mathrm{C} 14-\mathrm{C} 15 & 167.90(12) \\ \mathrm{C} 13-\mathrm{C} 14-\mathrm{C} 15-\mathrm{C} 16 & -48.13(18) \\ \mathrm{C} 19-\mathrm{C} 14-\mathrm{C} 15-\mathrm{C} 16 & 70.66(16) \\ \mathrm{C} 8-\mathrm{C} 14-\mathrm{C} 15-\mathrm{C} 16 & -170.20(12) \\ \mathrm{C} 14-\mathrm{C} 15-\mathrm{C} 16-\mathrm{C} 17 & 56.47(17) \\ \mathrm{C} 15-\mathrm{C} 16-\mathrm{C} 17-\mathrm{C} 24 & 64.84(18) \\ \mathrm{C} 15-\mathrm{C} 16-\mathrm{C} 17-\mathrm{C} 18 & -57.23(17) \\ \mathrm{C} 15-\mathrm{C} 16-\mathrm{C} 17-\mathrm{C} 28 & 178.72(14) \\ \mathrm{C} 12-\mathrm{C} 13-\mathrm{C} 18-\mathrm{C} 17 & 130.96(17) \\ \mathrm{C} 14-\mathrm{C} 13-\mathrm{C} 18-\mathrm{C} 17 & -49.90(18) \\ \mathrm{C} 12-\mathrm{C} 13-\mathrm{C} 18-\mathrm{C} 25 & -101.75(19) \\ \mathrm{C} 14-\mathrm{C} 13-\mathrm{C} 18-\mathrm{C} 25 & 77.39(18) \\ \mathrm{C} 24-\mathrm{C} 17-\mathrm{C} 18-\mathrm{C} 13 & -68.80(17)\end{array}$




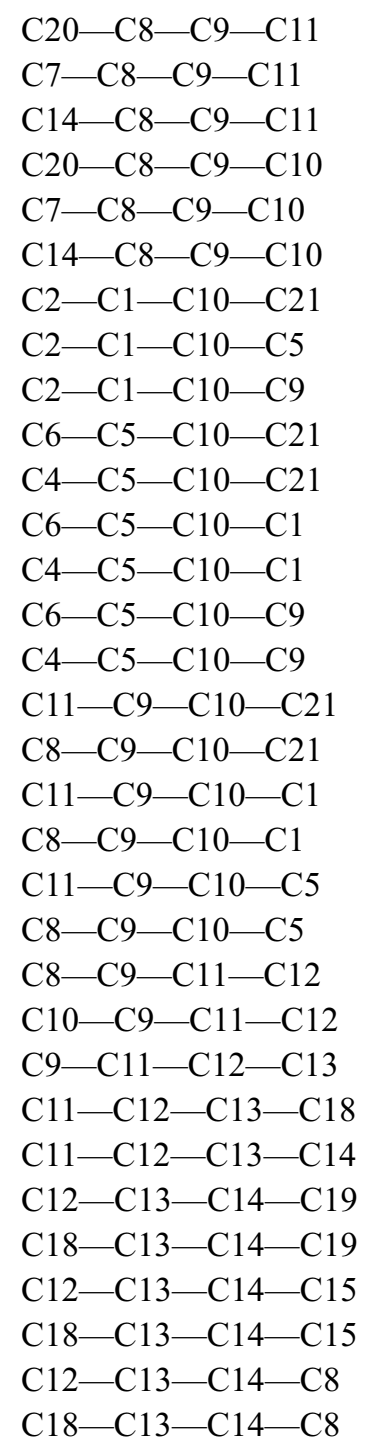

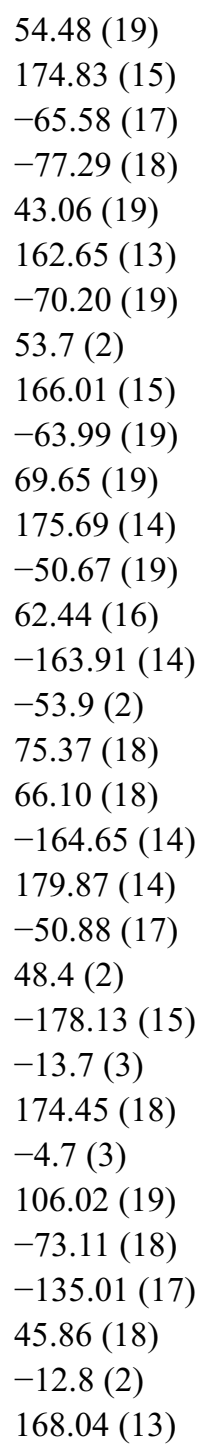

$54.48(19)$

$174.83(15)$

$-65.58(17)$

$-77.29(18)$

43.06 (19)

162.65 (13)

-70.20 (19)

53.7 (2)

$166.01(15)$

$-63.99(19)$

69.65 (19)

$175.69(14)$

-50.67 (19)

62.44 (16)

-163.91 (14)

$-53.9(2)$

75.37 (18)

66.10 (18)

-164.65 (14)

179.87 (14)

-50.88 (17)

48.4 (2)

$-178.13(15)$

$-13.7(3)$

174.45 (18)

-4.7 (3)

106.02 (19)

$-73.11(18)$

-135.01 (17)

45.86 (18)

$-12.8(2)$

168.04 (13)

$\begin{array}{ll}\mathrm{C} 16-\mathrm{C} 17-\mathrm{C} 18-\mathrm{C} 13 & 53.07(18) \\ \mathrm{C} 28-\mathrm{C} 17-\mathrm{C} 18-\mathrm{C} 13 & 177.31(13) \\ \mathrm{C} 24-\mathrm{C} 17-\mathrm{C} 18-\mathrm{C} 25 & 163.96(13) \\ \mathrm{C} 16-\mathrm{C} 17-\mathrm{C} 18-\mathrm{C} 25 & -74.17(17) \\ \mathrm{C} 28-\mathrm{C} 17-\mathrm{C} 18-\mathrm{C} 25 & 50.07(18) \\ \mathrm{C} 13-\mathrm{C} 14-\mathrm{C} 19-\mathrm{O} 3 & -47.5(2) \\ \mathrm{C} 15-\mathrm{C} 14-\mathrm{C} 19-\mathrm{O} 3 & -166.42(15) \\ \mathrm{C} 8-\mathrm{C} 14-\mathrm{C} 19-\mathrm{O} 3 & 72.86(18) \\ \mathrm{C} 13-\mathrm{C} 14-\mathrm{C} 19-\mathrm{O} 2 & 133.37(16) \\ \mathrm{C} 15-\mathrm{C} 14-\mathrm{C} 19-\mathrm{O} 2 & 14.4(2) \\ \mathrm{C} 8-\mathrm{C} 14-\mathrm{C} 19-\mathrm{O} 2 & -106.28(17) \\ \mathrm{C} 16-\mathrm{C} 17-\mathrm{C} 24-\mathrm{O} 4 & -149.64(17) \\ \mathrm{C} 18-\mathrm{C} 17-\mathrm{C} 24-\mathrm{O} 4 & -27.9(2) \\ \mathrm{C} 28-\mathrm{C} 17-\mathrm{C} 24-\mathrm{O} 4 & 91.1(2) \\ \mathrm{C} 16-\mathrm{C} 17-\mathrm{C} 24-\mathrm{O} 5 & 36.0(2) \\ \mathrm{C} 18-\mathrm{C} 17-\mathrm{C} 24-\mathrm{O} 5 & 157.70(16) \\ \mathrm{C} 28-\mathrm{C} 17-\mathrm{C} 24-\mathrm{O} 5 & -83.28(19) \\ \mathrm{C} 13-\mathrm{C} 18-\mathrm{C} 25-\mathrm{C} 30 & 61.30(19) \\ \mathrm{C} 17-\mathrm{C} 18-\mathrm{C} 25-\mathrm{C} 30 & -171.98(15) \\ \mathrm{C} 13-\mathrm{C} 18-\mathrm{C} 25-\mathrm{C} 26 & -177.17(14) \\ \mathrm{C} 17-\mathrm{C} 18-\mathrm{C} 25-\mathrm{C} 26 & -50.45(19) \\ \mathrm{C} 30-\mathrm{C} 25-\mathrm{C} 26-\mathrm{C} 27 & 174.28(17) \\ \mathrm{C} 18-\mathrm{C} 25-\mathrm{C} 26-\mathrm{C} 27 & 52.5(2) \\ \mathrm{C} 30-\mathrm{C} 25-\mathrm{C} 26-\mathrm{C} 29 & -63.2(2) \\ \mathrm{C} 18-\mathrm{C} 25-\mathrm{C} 26-\mathrm{C} 29 & 175.04(18) \\ \mathrm{C} 29-\mathrm{C} 26-\mathrm{C} 27-\mathrm{C} 28 & -179.62(17) \\ \mathrm{C} 25-\mathrm{C} 26-\mathrm{C} 27-\mathrm{C} 28 & -55.5(2) \\ \mathrm{C} 26-\mathrm{C} 27-\mathrm{C} 28-\mathrm{C} 17 & 56.3(2) \\ \mathrm{C} 24-\mathrm{C} 17-\mathrm{C} 28-\mathrm{C} 27 & -171.43(15) \\ \mathrm{C} 16-\mathrm{C} 17-\mathrm{C} 28-\mathrm{C} 27 & 70.3(2) \\ \mathrm{C} 18-\mathrm{C} 17-\mathrm{C} 28-\mathrm{C} 27 & -53.0(2) \\ & \end{array}$

$\mathrm{C} 16-\mathrm{C} 17-\mathrm{C} 18-\mathrm{C} 13$

53.07 (18)

$163.96(13)$

$-74.17(17)$

50.07 (18)

-47.5 (2)

$-166.42(15)$

$72.86(18)$

$133.37(16)$

14.4 (2)

$-106.28(17)$

-27.9 (2)

(2)

$157.70(16)$

$-83.28(19)$

61.30 (19)

$-171.98(15)$

$-177.17(14)$

-50.45 (19)

174.28 (17)

52.5 (2)

175.04 (18)

$179.62(17)$

56.3 (2)

$-171.43(15)$

-53.0 (2)

Hydrogen-bond geometry $\left(\AA,{ }^{\circ}\right)$

\begin{tabular}{lllll}
\hline$D-\mathrm{H} \cdots A$ & $D-\mathrm{H}$ & $\mathrm{H} \cdots A$ & $D \cdots A$ & $D-\mathrm{H} \cdots A$ \\
\hline $\mathrm{O} 4-\mathrm{H} 4 A \cdots \mathrm{O} 3^{\mathrm{i}}$ & $0.91(1)$ & $1.70(1)$ & $2.6046(18)$ & $172(5)$ \\
$\mathrm{O} 2-\mathrm{H} 19 A \cdots \mathrm{O} 5^{\mathrm{ii}}$ & $0.80(4)$ & $1.89(4)$ & $2.6702(18)$ & $165(4)$ \\
$\mathrm{C} 7-\mathrm{H} 7 A \cdots \mathrm{O} 2$ & 0.99 & 2.54 & $3.232(2)$ & 127 \\
$\mathrm{C} 9-\mathrm{H} 9 A \cdots \mathrm{O} 3$ & 1.00 & 2.18 & $3.009(2)$ & 139 \\
$\mathrm{C} 28-\mathrm{H} 28 B \cdots \mathrm{O} 1^{i i i}$ & 0.99 & 2.49 & $3.477(3)$ & 173 \\
$\mathrm{C} 29-\mathrm{H} 29 A \cdots \mathrm{O} 4{ }^{\text {ii }}$ & 0.98 & 2.57 & $3.497(3)$ & 158 \\
$\mathrm{C} 30-\mathrm{H} 30 A \cdots \mathrm{O} 3$ & 0.98 & 2.58 & $3.221(3)$ & 123 \\
\hline
\end{tabular}

Symmetry codes: (i) $x+1, y, z$; (ii) $x-1, y, z$; (iii) $x+1, y-1, z$; (iv) $-x+2, y-1 / 2,-z+1$. 